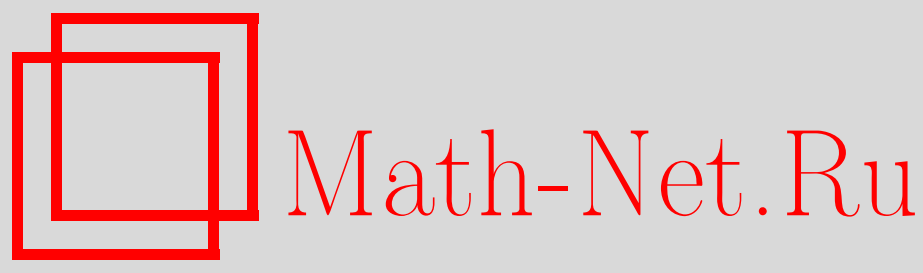

В. В. Дякин, О. В. Кудряшова, В. Я. Раевский, Один подход к решению основного уравнения магнитостатики для случая неоднородных магнетиков, ТМФ, 2016, том 187, номер 1, 88-103

DOI: https://doi.org/10.4213/tmf8956

Использование Общероссийского математического портала Math-Net.Ru подразумевает, что вы прочитали и согласны с пользовательским соглашением http://www . mathnet.ru/rus/agreement

Параметры загрузки:

IP : 35.173 .137 .237

26 апреля 2023 г., 06:06:22

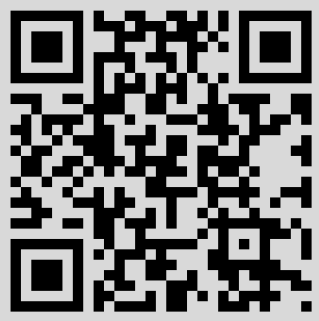




\title{
ОДИН ПОДХОД К РЕШЕНИЮ ОСНОВНОГО УРАВНЕНИЯ МАГНИТОСТАТИКИ ДЛЯ СЛУЧАЯ НЕОДНОРОДНЫХ МАГНЕТИКОВ
}

\begin{abstract}
Предлагается общий подход к решению основного уравнения магнитостатики в случае неоднородных магнитных тел, когда их магнитная проницаемость зависит от координат. Поиск напряженности результирующего поля сводится к определению двух скалярных функций из системы, содержащей интегральное и дифференциальное уравнения для этих функций с определенными граничными условиями. С помощью этого подхода получено аналитическое решение магнитостатического уравнения для шара с модельной магнитной проницаемостью в произвольном внешнем поле.
\end{abstract}

Ключевые слова: интегро-дифференциальное уравнение магнитостатики, сферические функции, разложение Вейля.

DOI: $10.4213 / \operatorname{tmf} 8956$

\section{1. ВВЕДЕНИЕ}

Для решения многих практических задач из области магнетизма (например, задач неразрушающего магнитного контроля) актуальной является проблема создания обоснованных алгоритмов аналитического или численного решения задач магнитостатики применительно к однородным или неоднородным магнитным телам различной формы с возможными инородными включениями. Для решения подобных задач мы исходим из так называемого основного уравнения магнитостатики (см., например, книгу [1]):

$$
\mathbf{H}(\mathbf{r})-\nabla \operatorname{div} \int_{\Omega} \frac{\left(\mu\left(\mathbf{r}^{\prime}\right)-1\right) \mathbf{H}\left(\mathbf{r}^{\prime}\right)}{4 \pi\left|\mathbf{r}-\mathbf{r}^{\prime}\right|} d \mathbf{r}^{\prime}=\mathbf{H}^{0}(\mathbf{r}), \quad \mathbf{r} \in \mathbb{R}^{3} \backslash S .
$$

Работа выполнена при поддержке программы Президиума РАН (проект № 15-17-2-54).

* Институт физики металлов УрО РАН, Екатеринбург, Россия.

E-mail: ovu@imp.uran.ru, ravskii@mail.ru

${ }^{\dagger}$ Уральский федеральный университет им. первого Президента России Б. Н. Ельцина, Екатеринбург, Россия. E-mail: kudryaschova.olga.valeryevna@yandex.ru 
Это уравнение эквивалентно системе уравнений Максвелла для случая магнитостатики и связывает искомую напряженность результирующего магнитного поля $\mathbf{H}(\mathbf{r})=\left\{H_{x}(\mathbf{r}), H_{y}(\mathbf{r}), H_{z}(\mathbf{r})\right\}$ в произвольной точке пространства $\mathbf{r}=(x, y, z)$ (не лежащей на границе магнетика) с заданным полем $\mathbf{H}^{0}(\mathbf{r})=\left\{H_{x}^{0}(\mathbf{r}), H_{y}^{0}(\mathbf{r}), H_{z}^{0}(\mathbf{r})\right\}$ внешнего источника. В данном уравнении $\Omega$ есть область в пространстве $\mathbb{R}^{3}$, ограниченная поверхностью $S$ и занятая исследуемым магнетиком с заданной магнитной проницаемостью $\mu(\mathbf{r})$, зависящей в общем случае от координат (неоднородный магнетик). Уравнение (1.1) справедливо для модели, в которой намагниченность магнетика $\mathbf{M}(\mathbf{r})$ связана с напряженностью $\mathbf{H}(\mathbf{r})$ соотношением $\mathbf{M}(\mathbf{r})=(\mu(\mathbf{r})-1) \mathbf{H}(\mathbf{r})$. Это соотношение является важным частным случаем общего материального уравнения классической магнитостатики и широко используется в задачах магнитного неразрушающего контроля изделий из технических сталей с мелкодисперсной структурой. В пользу адекватности указанной модели в задачах магнитного неразрушающего контроля говорит и тот факт, что внешнее поле, в которое помещается изделие, является стационарным (перемагничивания нет), а потому явление гистерезиса отсутствует.

Для определения из уравнения (1.1) напряженности результирующего поля $\mathbf{H}(\mathbf{r})$ как внутри магнетика (в этом случае обозначим напряженность $\mathbf{H}^{\mathrm{i}}(\mathbf{r})$ ), так и вне его (обозначим напряженность $\mathbf{H}^{\mathrm{e}}(\mathbf{r})$ ) поступают следующим образом. Сначала в соотношении (1.1) полагают, что $\mathbf{r} \in \Omega$, и получают уравнение для поля $\mathbf{H}^{\mathrm{i}}(\mathbf{r})$ внутри магнетика:

$$
\mathbf{H}^{\mathrm{i}}(\mathbf{r})-\nabla \operatorname{div} \int_{\Omega} \frac{\left(\mu\left(\mathbf{r}^{\prime}\right)-1\right) \mathbf{H}^{\mathrm{i}}\left(\mathbf{r}^{\prime}\right)}{4 \pi\left|\mathbf{r}-\mathbf{r}^{\prime}\right|} d \mathbf{r}^{\prime}=\mathbf{H}^{0}(\mathbf{r}), \quad \mathbf{r} \in \Omega .
$$

После решения этого уравнения относительно $\mathbf{H}^{\mathrm{i}}(\mathbf{r})$ в соотношении (1.1) полагают $\mathbf{r} \notin \Omega$, откуда вытекает, что формула для вычисления результирующего поля $\mathbf{H}^{\mathrm{e}}(\mathbf{r})$ вне магнетика имеет вид

$$
\mathbf{H}^{\mathrm{e}}(\mathbf{r})=\mathbf{H}^{0}(\mathbf{r})+\nabla \operatorname{div} \int_{\Omega} \frac{\left(\mu\left(\mathbf{r}^{\prime}\right)-1\right) \mathbf{H}^{\mathrm{i}}\left(\mathbf{r}^{\prime}\right)}{4 \pi\left|\mathbf{r}-\mathbf{r}^{\prime}\right|} d \mathbf{r}^{\prime}, \quad \mathbf{r} \notin \Omega .
$$

Поэтому основной проблемой является создание эффективных аналитических или численных методов решения уравнения (1.2). Доказано (см., например, [2]-[6]), что уравнение (1.2), рассматриваемое в вещественном гильбертовом пространстве $\mathbf{L}_{2}(\Omega)$ вектор-функций $\mathbf{M}(\mathbf{r})$ с (конечной) нормой $\|\mathbf{M}\|^{2}=\int_{\Omega}|\mathbf{M}(\mathbf{r})|^{2} d \mathbf{r}$ и скалярным произведением $(\mathbf{M}, \mathbf{N})=\int_{\Omega} \mathbf{M}(\mathbf{r}) \cdot \mathbf{N}(\mathbf{r}) d \mathbf{r}$, имеет единственное решение для ограниченных и неограниченных областей $\Omega$.

Ранее для задач неразрушающего магнитного контроля изделий были разработаны разнообразные методы аналитического и численного решения уравнения (1.2) для ограниченных и неограниченных магнетиков с внутренними полостями (дефектами) различной формы (см., например, [7]-[11]). Успешность решения этих задач во многом определялась тем обстоятельством, что магнитная проницаемость магнетика $\mu(\mathbf{r})$ предполагалась постоянной (или кусочно-постоянной), что значительно облегчало поиск решения уравнения (1.2), поскольку ограничивало этот поиск гармоническими векторными полями. В настоящей работе предлагается подход к решению уравнения (1.2) для случая изотропных, но неоднородных магнетиков, когда их магнитная проницаемость $\mu(\mathbf{r})$ является произвольной функцией от координат. 
Поиск напряженности результирующего поля $\mathbf{H}^{\mathrm{i}}(\mathbf{r})$ сводится к определению двух скалярных функций из системы, содержащей интегральное и обыкновенное дифференциальное уравнения с определенными граничными условиями. В качестве важной иллюстрации предлагаемого подхода получены аналитические формулы для напряженности результирующего поля $\mathbf{H}^{\mathrm{i}}(\mathbf{r})$ внутри и $\mathbf{H}^{\mathrm{e}}(\mathbf{r})$ вне шарового магнетика с модельной (зависящей от координат) магнитной проницаемостью $\mu(\mathbf{r})$ в произвольном внешнем поле $\mathbf{H}^{0}(\mathbf{r})$.

\section{2. ВЫВОД ОСНОВНЫХ УРАВНЕНИЙ}

В дальнейшем нам понадобится так называемое разложение Вейля пространства $\mathbf{L}_{2}(\Omega)$ на ортогональную сумму трех подпространств [12]:

$$
\mathbf{L}_{2}(\Omega)=\mathbf{U}(\Omega) \oplus \mathbf{G}(\Omega) \oplus \mathbf{J}(\Omega),
$$

где $\mathbf{U}(\Omega)$ - подпространство градиентов гармонических в $\Omega$ функций из соболевского пространства $H^{1}(\Omega), \mathbf{G}(\Omega)$ - подпространство градиентов функций из $H^{1}(\Omega)$, обращающихся в нуль на границе $S$ области $\Omega$, а $\mathbf{J}(\Omega)$ - замыкание в $\mathbf{L}_{2}(\Omega)$ линейного многообразия гладких соленоидальных в $\Omega$ вектор-функций с нулевой нормальной составляющей на границе $S$ :

$$
\begin{gathered}
\mathbf{U}(\Omega)=\left\{\nabla \eta: \Delta \eta(\mathbf{r})=0, \mathbf{r} \in \Omega, \eta \in H^{1}(\Omega)\right\}, \\
\mathbf{G}(\Omega)=\left\{\nabla \psi: \psi \in H^{1}(\Omega),\left.\psi\right|_{S}=0\right\} \\
\mathbf{J}(\Omega)=\overline{\left\{\mathbf{P}: \operatorname{div} \mathbf{P}(\mathbf{r})=0, \mathbf{r} \in \Omega,\left.P_{n}\right|_{S}=0\right\}} .
\end{gathered}
$$

Вернемся к уравнению (1.2). Его правая часть $\mathbf{H}^{0}(\mathbf{r})$ представляет собой напряженность известного внешнего поля, созданного сторонними источниками тока. Поскольку предполагается, что область этих токов не пересекается с областью магнетика $\Omega$, согласно системе уравнений Максвелла $\operatorname{div} \mathbf{H}^{0}(\mathbf{r})=0, \operatorname{rot} \mathbf{H}^{0}(\mathbf{r})=0$ в $\Omega$, а потому $\mathbf{H}^{0}(\mathbf{r})$ есть бесконечно гладкое гармоническое поле в $\Omega$, которое имеет вид

$$
\mathbf{H}^{0}(\mathbf{r})=\nabla \eta^{0}(\mathbf{r}), \quad \Delta \eta^{0}(\mathbf{r})=0, \quad \mathbf{r} \in \Omega .
$$

Докажем прежде всего, что если достаточно гладкая функция $\mathbf{H}^{\mathrm{i}}(\mathbf{r})$ является решением уравнения (1.2), она необходимо удовлетворяет следующим соотношениям:

$$
\operatorname{rot} \mathbf{H}^{\mathrm{i}}(\mathbf{r})=\mathbf{0}, \quad \operatorname{div}\left[\mu(\mathbf{r}) \mathbf{H}^{\mathrm{i}}(\mathbf{r})\right]=0, \quad \mathbf{r} \in \Omega .
$$

Для доказательства первого соотношения достаточно применить операцию rot к обеим частям (1.2) и учесть (2.5). Для доказательства второго соотношения в (2.6) введем вспомогательную функцию

$$
W(\mathbf{r}):=-\operatorname{div} \int_{\Omega} \frac{\left(\mu\left(\mathbf{r}^{\prime}\right)-1\right) \mathbf{H}^{\mathrm{i}}\left(\mathbf{r}^{\prime}\right)}{4 \pi\left|\mathbf{r}-\mathbf{r}^{\prime}\right|} d \mathbf{r}^{\prime},
$$

которую (после внесения операции div под знак интеграла и интегрирования по частям) можно представить в виде

$$
W(\mathbf{r})=\int_{S} \frac{\left(\mu\left(\mathbf{r}^{\prime}\right)-1\right) H_{n^{\prime}}^{\mathrm{i}}\left(\mathbf{r}^{\prime}\right)}{4 \pi\left|\mathbf{r}-\mathbf{r}^{\prime}\right|} d \mathbf{r}^{\prime}-\int_{\Omega} \frac{\operatorname{div}\left[\left(\mu\left(\mathbf{r}^{\prime}\right)-1\right) \mathbf{H}^{\mathrm{i}}\left(\mathbf{r}^{\prime}\right)\right]}{4 \pi\left|\mathbf{r}-\mathbf{r}^{\prime}\right|} d \mathbf{r}^{\prime},
$$


где нижний индекс $n^{\prime}$ означает переход к нормальной составляющей по внешней нормали в текущей точке интегрирования $\mathbf{r}^{\prime}$. Учитывая свойства потенциала простого слоя и объемного потенциала, из (2.8) имеем

$$
\Delta W(\mathbf{r})=\operatorname{div}\left[(\mu(\mathbf{r})-1) \mathbf{H}^{\mathrm{i}}(\mathbf{r})\right], \quad \mathbf{r} \in \Omega .
$$

Записывая уравнение (1.2) в виде

$$
\mathbf{H}^{\mathrm{i}}(\mathbf{r})+\nabla W(\mathbf{r})=\mathbf{H}^{0}(\mathbf{r})
$$

и вычисляя дивергенцию от обеих частей, с учетом (2.5) и приведенного выше выражения для $\Delta W(\mathbf{r})$ получим второе соотношение в (2.6). Отметим, что это соотношение является отражением известного физического свойства - соленоидальности индукции магнитного поля, но здесь важно то, что оно является необходимым свойством решения уравнения (1.2).

Из первого соотношения в (2.6) следует, что в представлении решения $\mathbf{H}^{\mathrm{i}}(\mathbf{r})$ уравнения (1.2) в виде суммы трех функций в соответствии с разложением Вейля (2.1) обращается в нуль последнее слагаемое, соответствующее проекции $\mathbf{H}^{\mathrm{i}}(\mathbf{r})$ на подпространство $\mathbf{J}(\Omega)$. Таким образом, решение уравнения (1.2) можно представить в виде

$$
\mathbf{H}^{\mathrm{i}}(\mathbf{r})=\nabla \eta(\mathbf{r})+\nabla \psi(\mathbf{r})
$$

причем

$$
\left.\psi\right|_{S}=0, \quad \Delta \eta(\mathbf{r})=0, \quad \mathbf{r} \in \Omega .
$$

Поэтому будем искать решение уравнения (1.2) в виде (2.10) с условием (2.11) и получим уравнения для определения скалярных функций $\psi$ и $\eta$.

При подстановке (2.10) в первое соотношение в (2.6) с учетом (2.11) легко получить следующую связь искомых функций $\psi$ и $\eta$ :

$$
\Delta \psi(\mathbf{r})+(\nabla \psi(\mathbf{r})+\nabla \eta(\mathbf{r})) \cdot \nabla \ln \mu(\mathbf{r})=0, \quad \mathbf{r} \in \Omega .
$$

Далее преобразуем выражения в числителях под интегралами в (2.8) с учетом (2.10). В поверхностном интеграле имеем

$$
(\mu(\mathbf{r})-1) H_{n}^{i}(\mathbf{r})=(\mu(\mathbf{r})-1)\left(\frac{\partial \psi(\mathbf{r})}{\partial n}+\frac{\partial \eta(\mathbf{r})}{\partial n}\right),
$$

в объемном интеграле с учетом второго соотношения в (2.6) имеем

$$
\operatorname{div}\left[(\mu(\mathbf{r})-1) \mathbf{H}^{\mathrm{i}}(\mathbf{r})\right]=-\operatorname{div} \mathbf{H}^{\mathrm{i}}(\mathbf{r})=-\Delta \psi(\mathbf{r}) .
$$

Подставляя полученные выражения в (2.8), придем к формуле

$$
W(\mathbf{r})=\int_{S} \frac{\left(\mu\left(\mathbf{r}^{\prime}\right)-1\right)\left(\partial \psi\left(\mathbf{r}^{\prime}\right) / \partial n^{\prime}+\partial \eta\left(\mathbf{r}^{\prime}\right) / \partial n^{\prime}\right)}{4 \pi\left|\mathbf{r}-\mathbf{r}^{\prime}\right|} d \mathbf{r}^{\prime}+\int_{\Omega} \frac{\Delta \psi\left(\mathbf{r}^{\prime}\right)}{4 \pi\left|\mathbf{r}-\mathbf{r}^{\prime}\right|} d \mathbf{r}^{\prime} .
$$

Учитывая основную интегральную формулу Грина (см., например, книги [13], [14]), представим $\psi(\mathbf{r})$ для $\mathbf{r} \in \Omega$ в виде

$$
\psi(\mathbf{r})=\int_{S}\left(\frac{\partial \psi\left(\mathbf{r}^{\prime}\right) / \partial n^{\prime}}{4 \pi\left|\mathbf{r}-\mathbf{r}^{\prime}\right|}-\psi\left(\mathbf{r}^{\prime}\right) \frac{\partial}{\partial n^{\prime}} \frac{1}{4 \pi\left|\mathbf{r}-\mathbf{r}^{\prime}\right|}\right) d \mathbf{r}^{\prime}-\int_{\Omega} \frac{\Delta \psi\left(\mathbf{r}^{\prime}\right)}{4 \pi\left|\mathbf{r}-\mathbf{r}^{\prime}\right|} d \mathbf{r}^{\prime} .
$$


Учитывая (2.11), выразим объемный интеграл из (2.14) и подставим его в (2.13), после чего имеем

$$
W(\mathbf{r})=-\psi(\mathbf{r})+\int_{S} \frac{\left.\mu\left(\mathbf{r}^{\prime}\right) \partial \psi\left(\mathbf{r}^{\prime}\right) / \partial n^{\prime}+\left(\mu\left(\mathbf{r}^{\prime}\right)-1\right) \partial \eta\left(\mathbf{r}^{\prime}\right) / \partial n^{\prime}\right)}{4 \pi\left|\mathbf{r}-\mathbf{r}^{\prime}\right|} d \mathbf{r}^{\prime} .
$$

Подставляя $W(\mathbf{r})$ из $(2.15)$ в (2.9), с учетом (2.5) и (2.10) окончательно запишем соотношение, связывающее искомые функции $\psi$ и $\eta$ :

$$
\nabla \eta(\mathbf{r})+\nabla \int_{S} \frac{\left.\mu\left(\mathbf{r}^{\prime}\right) \partial \psi\left(\mathbf{r}^{\prime}\right) / \partial n^{\prime}+\left(\mu\left(\mathbf{r}^{\prime}\right)-1\right) \partial \eta\left(\mathbf{r}^{\prime}\right) / \partial n^{\prime}\right)}{4 \pi\left|\mathbf{r}-\mathbf{r}^{\prime}\right|} d \mathbf{r}^{\prime}=\nabla \eta^{0}(\mathbf{r}) .
$$

Таким образом, учитывая выражения (2.10)-(2.12) и (2.16), получим следующую компактную схему предлагаемого подхода для поиска решения $\mathbf{H}^{\mathrm{i}}(\mathbf{r})$ уравнения (1.2) в случае зависимости магнитной проницаемости от координат: для $r \in \Omega$ имеем систему уравнений

$$
\begin{gathered}
\mathbf{H}^{\mathrm{i}}(\mathbf{r})=\nabla \eta(\mathbf{r})+\nabla \psi(\mathbf{r}), \\
\nabla \eta(\mathbf{r})+\nabla \int_{S} \frac{\left.\mu\left(\mathbf{r}^{\prime}\right) \partial \psi\left(\mathbf{r}^{\prime}\right) / \partial n^{\prime}+\left(\mu\left(\mathbf{r}^{\prime}\right)-1\right) \partial \eta\left(\mathbf{r}^{\prime}\right) / \partial n^{\prime}\right)}{4 \pi\left|\mathbf{r}-\mathbf{r}^{\prime}\right|} d \mathbf{r}^{\prime}=\nabla \eta^{0}(\mathbf{r}), \\
\Delta \psi(\mathbf{r})+(\nabla \psi(\mathbf{r})+\nabla \eta(\mathbf{r})) \cdot \nabla \ln \mu(\mathbf{r})=0,\left.\quad \psi(\mathbf{r})\right|_{S}=0, \quad \Delta \eta(\mathbf{r})=0 .
\end{gathered}
$$

\section{3. СЛУЧАЙ ШАРОВОЙ ФОРМЫ МАГНЕТИКА}

В качестве важного примера применим указанный подход (2.17) для решения уравнения (1.2) в случае, когда область магнетика $\Omega$ является шаром произвольного радиуса $r_{0}$. Начало декартовой системы координат выберем в центре этого шара, внешнее поле $\mathbf{H}^{0}(\mathbf{r})$ по-прежнему считаем произвольным, а на магнитную проницаемость наложим пока единственное требование сферической симметричности: $\mu(\mathbf{r})=\mu(r)$, где $r=|\mathbf{r}|$. В дальнейшем мы часто переходим от декартовой к сферической системе координат по известным формулам $x=r \sin \theta \cos \varphi, y=r \sin \theta \sin \varphi$, $z=r \cos \theta$, поэтому для компактности введем следующее обозначение: $\left.F(\mathbf{r})\right|_{\mathrm{sph}}$ означает выражение, получающееся после перехода в выражении $F(\mathbf{r})$ от декартовых координат $\mathbf{r}=(x, y, z)$ к сферическим $(r, \theta, \varphi)$. Для определения напряженности результирующего поля в описанной ситуации будем искать функции $\psi(\mathbf{r})$ и $\eta(\mathbf{r})$ из условий (2.17), точнее, вид этих функций после перехода к сферической системе координат:

$$
\psi(r, \theta, \varphi):=\left.\psi(\mathbf{r})\right|_{\mathrm{sph}}, \quad \eta(r, \theta, \varphi):=\left.\eta(\mathbf{r})\right|_{\mathrm{sph}} .
$$

Далее нами используется система сферических функций $\left\{Y_{l m}(\theta, \varphi)\right\}, l=0,1,2, \ldots$, $m=-l,-l+1, \ldots, l-1, l$, которая (при некоторых разночтениях в литературе) здесь определяется формулами (см., например, [15]):

$$
\begin{gathered}
Y_{l m}(\theta, \varphi)=(-1)^{m} \sqrt{\frac{2 l+1}{4 \pi} \frac{(l-m) !}{(l+m) !}} P_{l}^{m}(\cos \theta) e^{\mathrm{im} \varphi}, \quad m \geqslant 0, \\
P_{l}^{m}(t)=\left(1-t^{2}\right)^{m / 2} \frac{d^{m} P_{l}(t)}{d t^{m}} \quad(\text { присоединенные полиномы Лежандра), } \\
P_{l}(t)=\frac{1}{2^{l} l !} \frac{d^{l}}{d t^{l}}\left(t^{2}-1\right)^{l} \quad \text { (полиномы Лежандра), } \\
Y_{l,-m}(\theta, \varphi)=(-1)^{m} Y_{l m}(\theta,-\varphi)=(-1)^{m} Y_{l m}^{*}(\theta, \varphi), \quad m>0,
\end{gathered}
$$


где звездочка в верхнем индексе означает переход к комплексному сопряжению. В дальнейшем нам понадобится базисное свойство системы $\left\{Y_{l m}(\theta, \varphi)\right\}$ в пространстве квадратично-суммируемых на единичной сфере функций, их ортонормированность,

$$
\int_{0}^{2 \pi} \int_{0}^{\pi} Y_{l m}(\theta, \varphi) Y_{l^{\prime} m^{\prime}}^{*}(\theta, \varphi) \sin \theta d \theta d \varphi=\delta_{l l^{\prime}} \delta_{m m^{\prime}}, \quad \delta_{n k}:= \begin{cases}0, & n \neq k \\ 1, & n=k\end{cases}
$$

а также известное разложение (см., например, [15]) вида

$$
\left.\frac{1}{4 \pi\left|\mathbf{r}-\mathbf{r}^{\prime}\right|}\right|_{\mathrm{sph}}=\frac{1}{r_{>}} \sum_{l=0}^{\infty} \frac{1}{2 l+1}\left(\frac{r_{<}}{r_{>}}\right)^{l} \sum_{m=-l}^{l} Y_{l m}(\theta, \varphi) Y_{l m}^{*}\left(\theta^{\prime}, \varphi^{\prime}\right)
$$

где $r_{<}=\min \left(r^{\prime}, r\right), r_{>}=\max \left(r^{\prime}, r\right)$.

Функция $\eta^{0}(\mathbf{r})$ в $(2.5)$ является гармонической в $\Omega$ и ограниченной в начале координат известной функцией, а потому функцию $\eta^{0}(r, \theta, \varphi):=\left.\eta^{0}(\mathbf{r})\right|_{\mathrm{sph}}$ можно представить в виде

$$
\eta^{0}(r, \theta, \varphi)=\sum_{l=1}^{\infty} \sum_{m=-l}^{l} \eta_{l m}^{0} r^{l} Y_{l m}(\theta, \varphi)
$$

где коэффициенты разложения $\left\{\eta_{l m}^{0}\right\}$ считаются известными. Внешнее суммирование в (3.5) начато с $l=1$, а не с $l=0$, поскольку слагаемое, соответствующее $l=0$, является константой и его можно опустить, так как $\eta^{0}(\mathbf{r})$ в $(2.5)$ определяется соотношением $\mathbf{H}^{0}(\mathbf{r})=\nabla \eta^{0}(\mathbf{r})$ с точностью до аддитивной постоянной. Поскольку функция $\eta(\mathbf{r})$ в $(2.17)$ также является гармонической в $\Omega$ и конечной в начале координат, искомая функция $\eta(r, \theta, \varphi)$ в $(3.1)$ представляется в виде ряда

$$
\eta(r, \theta, \varphi)=\sum_{l=0}^{\infty} \sum_{m=-l}^{l} \eta_{l m} r^{l} Y_{l m}(\theta, \varphi)
$$

Отметим, что, поскольку в выражение для поля $\mathbf{H}^{\mathrm{i}}(\mathbf{r})$ в $(2.17)$ функция $\eta(\mathbf{r})$ входит под знаком градиента, ее можно определить с точностью до аддитивной произвольной постоянной. Поэтому значение $\eta_{00}$ в (3.6) может быть произвольным. Для определенности будем считать $\eta_{00}=0$.

Аналогично, из указанного выше базисного свойства сферических функций выведем, что при каждом фиксированном $r$ искомая функция $\psi(r, \theta, \varphi)$ в (3.1) представляется в виде разложения по системе сферических функций с коэффициентами $\left\{\psi_{l m}(r)\right\}$, зависящими от $r$ :

$$
\psi(r, \theta, \varphi)=\sum_{l=0}^{\infty} \sum_{m=-l}^{l} \psi_{l m}(r) Y_{l m}(\theta, \varphi)
$$

причем из условия $\left.\psi(\mathbf{r})\right|_{S}=0$ в $(2.17)$ следует, что для функций $\left\{\psi_{l m}(r)\right\}$ должно выполняться соотношение

$$
\psi_{l m}\left(r_{0}\right)=0, \quad l=0,1,2, \ldots, \quad m=-l,-l+1, \ldots, l-1, l .
$$


Таким образом, для поиска функций $\psi(r, \theta, \varphi)$ и $\eta(r, \theta, \varphi)$ достаточно в представлениях (3.6) и (3.7) найти наборы чисел $\left\{\eta_{l m}\right\}$ и функций $\left\{\psi_{l m}(r)\right\}$, для которых выполняется условие (3.8).

Зафиксируем во втором уравнении (2.17) вектор $\mathbf{r} \in \Omega$ и умножим (скалярно) обе части уравнения на вектор $\mathbf{n}:=\mathbf{r} /|\mathbf{r}|$, получим выражение

$$
\frac{\partial \eta(\mathbf{r})}{\partial n}+\frac{\partial V(\mathbf{r})}{\partial n}=\frac{\partial \eta^{0}(\mathbf{r})}{\partial n}
$$

где для удобства введена функция

$$
V(\mathbf{r}):=\int_{S} \frac{\left.\mu\left(\mathbf{r}^{\prime}\right) \partial \psi\left(\mathbf{r}^{\prime}\right) / \partial n^{\prime}+\left(\mu\left(\mathbf{r}^{\prime}\right)-1\right) \partial \eta\left(\mathbf{r}^{\prime}\right) / \partial n^{\prime}\right)}{4 \pi\left|\mathbf{r}-\mathbf{r}^{\prime}\right|} d \mathbf{r}^{\prime} .
$$

Переходя в (3.9) к сферической системе координат, учтем, что в данном случае $\partial / \partial n$ переходит в $\partial / \partial r$ :

$$
\frac{\partial \eta(r, \theta, \varphi)}{\partial r}+\frac{\partial V(r, \theta, \varphi)}{\partial r}=\frac{\partial \eta^{0}(r, \theta, \varphi)}{\partial r},
$$

где $V(r, \theta, \varphi):=\left.V(\mathbf{r})\right|_{\mathrm{sph}}$. Найдем выражение для $V(r, \theta, \varphi)$, переходя в формуле (3.10) к сферической системе координат и используя представления (3.6), (3.7) и разложение $(3.4)$ :

$$
\begin{aligned}
V(r, \theta, \varphi)= & \int_{0}^{2 \pi} \int_{0}^{\pi}\left(\mu^{0} \sum_{l=0}^{\infty} \sum_{m=-l}^{l} \psi_{l m}^{\prime}\left(r_{0}\right) Y_{l m}\left(\theta^{\prime}, \varphi^{\prime}\right)+\right. \\
& \left.+\left(\mu^{0}-1\right) \sum_{l=0}^{\infty} \sum_{m=-l}^{l} l \eta_{l m} r_{0}^{l-1} Y_{l m}\left(\theta^{\prime}, \varphi^{\prime}\right)\right) \times \\
& \times\left(\frac{1}{r_{0}} \sum_{l=0}^{\infty} \sum_{m=-l}^{l} \frac{1}{2 l+1} \frac{r^{l}}{r_{0}^{l}} Y_{l m}(\theta, \varphi) Y_{l m}^{*}\left(\theta^{\prime}, \varphi^{\prime}\right)\right) r_{0}^{2} \sin \theta^{\prime} d \theta^{\prime} d \varphi^{\prime},
\end{aligned}
$$

где $\mu^{0}=\mu\left(r_{0}\right)$ - постоянное значение магнитной проницаемости на границе $S$ области $\Omega$. Учитывая ортонормированность сферических функций (3.3), имеем

$$
V(r, \theta, \varphi)=\sum_{l=0}^{\infty} \sum_{m=-l}^{l} \frac{q_{l m}}{2 l+1} \frac{r^{l}}{r_{0}^{l-1}} Y_{l m}(\theta, \varphi),
$$

где

$$
q_{l m}:=\mu^{0} \psi_{l m}^{\prime}\left(r_{0}\right)+l\left(\mu^{0}-1\right) \eta_{l m} r_{0}^{l-1} .
$$

Вычисляя в выражениях $(3.5),(3.6)$ и (3.12) производные по $r$ от $\eta^{0}(r, \theta, \varphi), \eta(r, \theta, \varphi)$, $V(r, \theta, \varphi)$ соответственно и подставляя их в $(3.11)$, придем к следующему соотношению:

$$
\sum_{l=0}^{\infty} \sum_{m=-l}^{l} l\left(\eta_{l m}+\frac{q_{l m}}{(2 l+1) r_{0}^{l-1}}\right) r^{l-1} Y_{l m}(\theta, \varphi)=\sum_{l=1}^{\infty} \sum_{m=-l}^{l} l \eta_{l m}^{0} r^{l-1} Y_{l m}(\theta, \varphi) .
$$

Учитывая базисное свойство системы сферических функций и равенство (3.13), получим выражение

$$
\left(l \mu^{0}+l+1\right) \eta_{l m}+\frac{\mu^{0}}{r_{0}^{l-1}} \psi_{l m}^{\prime}\left(r_{0}\right)=(2 l+1) \eta_{l m}^{0}, \quad l=1,2, \ldots, \quad m=-l,-l+1, \ldots, l .
$$


Именно такие ограничения налагает уравнение во второй строке системы (2.17) на искомые наборы чисел $\left\{\eta_{l m}\right\}$ и функций $\left\{\psi_{l m}(r)\right\}$.

Рассмотрим теперь уравнение в третьей строке (2.17), в котором также перейдем к сферическим координатам. Применяя выражение

$$
\left.\Delta \psi(\mathbf{r})\right|_{\mathrm{sph}}=\frac{1}{r^{2}}\left(\frac{\partial}{\partial r}\left(r^{2} \frac{\partial \psi}{\partial r}\right)+\frac{1}{\sin \theta} \frac{\partial}{\partial \theta}\left(\sin \theta \frac{\partial \psi}{\partial \theta}\right)+\frac{1}{\sin ^{2} \theta} \frac{\partial^{2} \psi}{\partial \varphi^{2}}\right)
$$

для оператора Лапласа в сферической системе координат к представлению (3.7) и учитывая, что сферические функции удовлетворяют известному соотношению (см., например, книгу [16])

$$
\frac{1}{\sin \theta} \frac{\partial}{\partial \theta}\left(\sin \theta \frac{\partial Y_{l m}(\theta, \varphi)}{\partial \theta}\right)+\frac{1}{\sin ^{2} \theta} \frac{\partial^{2} Y_{l m}(\theta, \varphi)}{\partial \varphi^{2}}+l(l+1) Y_{l m}(\theta, \varphi)=0,
$$

выпишем равенство

$$
\left.\Delta \psi(\mathbf{r})\right|_{\mathrm{sph}}=\sum_{l=0}^{\infty} \sum_{m=-l}^{l} r^{-2}\left(r^{2} \psi_{l m}^{\prime \prime}(r)+2 r \psi_{l m}^{\prime}(r)-l(l+1) \psi_{l m}(r)\right) Y_{l m}(\theta, \varphi) .
$$

Далее, учитывая представление градиента в сферической системе координат

$$
\left.\nabla U(\mathbf{r})\right|_{\mathrm{sph}}=\frac{\partial U}{\partial r} \mathbf{e}_{r}+\frac{1}{r \sin \theta} \frac{\partial U}{\partial \varphi} \mathbf{e}_{\varphi}+\frac{1}{r} \frac{\partial U}{\partial \theta} \mathbf{e}_{\theta}
$$

и вводя для краткости обозначение $f(r):=\ln \mu(r)$, выведем следующее равенство: $\left.\nabla \ln \mu(\mathbf{r})\right|_{\mathrm{sph}}=f^{\prime}(r) \mathbf{e}_{r}$. Поскольку оно содержит только компоненту $\mathbf{e}_{r}$, а в уравнение третьей строки системы (2.17) входит скалярное произведение, в выражениях $\left.\nabla \psi(\mathbf{r})\right|_{\mathrm{sph}}$ и $\left.\nabla \eta(r)\right|_{\mathrm{sph}}$ нам важны только компоненты $\mathbf{e}_{r}$. Применяя (3.16) к представлениям (3.6) и (3.7), получим

$$
\begin{aligned}
& \left.\nabla \eta(\mathbf{r})\right|_{\mathrm{sph}}=\sum_{l=0}^{\infty} \sum_{m=-l}^{l} l \eta_{l m} r^{l-1} Y_{l m}(\theta, \varphi) \mathbf{e}_{r}+\cdots, \\
& \left.\nabla \psi(\mathbf{r})\right|_{\mathrm{sph}}=\sum_{l=0}^{\infty} \sum_{m=-l}^{l} \psi_{l m}^{\prime}(r) Y_{l m}(\theta, \varphi) \mathbf{e}_{r}+\cdots
\end{aligned}
$$

Подставляя найденные выражения в уравнение третьей строки системы (2.17), запишем это уравнение в сферической системе координат:

$$
\begin{gathered}
\sum_{l=0}^{\infty} \sum_{m=-l}^{l}\left\{r^{-2}\left(r^{2} \psi_{l m}^{\prime \prime}(r)+2 r \psi_{l m}^{\prime}(r)-l(l+1) \psi_{l m}(r)\right)+\right. \\
\left.+\left(\psi_{l m}^{\prime}(r)+l \eta_{l m} r^{l-1}\right) f^{\prime}(r)\right\} Y_{l m}(\theta, \varphi)=0 .
\end{gathered}
$$

Учитывая базисное свойство системы сферических функций, заключаем, что числа $\left\{\eta_{l m}\right\}$ и функции $\left\{\psi_{l m}(r)\right\}$ должны удовлетворять дифференциальным уравнениям

$$
r^{2} \psi_{l m}^{\prime \prime}(r)+r\left(r f^{\prime}(r)+2\right) \psi_{l m}^{\prime}(r)-l(l+1) \psi_{l m}(r)=-l \eta_{l m} r^{l+1} f^{\prime}(r) .
$$


При $l=0$ (следовательно, $m=0)$ уравнение (3.17) принимает вид

$$
r^{2} \psi_{00}^{\prime \prime}(r)+r\left(r f^{\prime}(r)+2\right) \psi_{00}^{\prime}(r)=0 .
$$

Легко показать, что решение этого уравнения, ограниченное в нуле, необходимо имеет вид $\psi_{00}(r)=$ const. Из начального условия $(3.8)$ тогда следует, что $\psi_{00}(r)=0$.

Подводя итог вышесказанному, получим, что для решения уравнения (1.2) с помощью схемы (2.17) функции $\eta(\mathbf{r})$ и $\psi(\mathbf{r})$ представляются в виде разложений (3.6) и (3.7), в которых коэффициенты $\left\{\eta_{l m}\right\}$ и $\left\{\psi_{l m}(r)\right\}$ являются решением следующей системы:

$$
\begin{gathered}
r^{2} \psi_{l m}^{\prime \prime}(r)+r\left(r f^{\prime}(r)+2\right) \psi_{l m}^{\prime}(r)-l(l+1) \psi_{l m}(r)=-l \eta_{l m} r^{l+1} f^{\prime}(r) \\
\left(l \mu^{0}+l+1\right) \eta_{l m}+\frac{\mu^{0}}{r_{0}^{l-1}} \psi_{l m}^{\prime}\left(r_{0}\right)=(2 l+1) \eta_{l m}^{0} \\
\psi_{l m}\left(r_{0}\right)=0, \quad \psi_{00}(r)=0, \quad \eta_{00}=0
\end{gathered}
$$

где $l=1,2, \ldots, m=-l,-l+1, \ldots, l$. Отметим, что условия в последней строке $(3.18)$ позволяют начинать внешнее суммирование в (3.6) и (3.7) с $l=1$.

\section{4. АНАЛИТИЧЕСКОЕ РЕШЕНИЕ МАГНИТОСТАТИЧЕСКОГО УРАВНЕНИЯ ДЛЯ ЭКСПОНЕНЦИАЛЬНОЙ МОДЕЛИ МАГНИТНОЙ ПРОНИЦАЕМОСТИ}

Найти аналитическое решение дифференциального уравнения в (3.18) для произвольной функции $f(r)$, по всей видимости, нельзя. Поэтому для дальнейшего продвижения по пути поиска аналитических решений мы конкретизируем модель магнитной проницаемости, оставив внешнее поле $\mathbf{H}^{0}(\mathbf{r})$ по-прежнему произвольным. Положим $\mu(r)=c_{0} e^{\alpha r}$, где $c_{0}>0$ и $\alpha-$ произвольные параметры. Подобного вида модель может соответствовать магнитной проницаемости шарового изделия (например, шарика как элемента шарикоподшипника), подвергнутого поверхностной закалке или наклепу (равномерному обжатию). Тогда $f(r):=\ln \mu(r)=\ln c_{0}+\alpha r$, а потому дифференциальное уравнение в первой строке (3.18) имеет вид

$$
r^{2} \psi_{l m}^{\prime \prime}(r)+r(\alpha r+2) \psi_{l m}^{\prime}(r)-l(l+1) \psi_{l m}(r)=-l \alpha \eta_{l m} r^{l+1} .
$$

Это уравнение является линейным уравнением второго порядка. Для определения его общего решения найдем сначала общее решение соответствующего однородного уравнения:

$$
r^{2} \psi_{l m}^{\prime \prime}(r)+r(\alpha r+2) \psi_{l m}^{\prime}(r)-l(l+1) \psi_{l m}(r)=0 .
$$

Совершая в нем замену, будем искать функцию $y(r)$ такую, что $\psi(r)=r^{l} y(r)$ есть решение уравнения (4.2). После подстановки функции $\psi(r)$ такого вида в $(4.2)$ и некоторых преобразований получим уравнение $\left[r y^{\prime}(r)+(\alpha l r+2 l+1) y(r)\right]^{\prime}=0$, откуда следует уравнение $r y^{\prime}(r)+(\alpha l r+2 l+1) y(r)=A$, где $A-$ произвольная постоянная. Последнее уравнение есть линейное уравнение первого порядка. Решая его по стандартной схеме, нетрудно найти общее решение:

$$
y(r)=\frac{A}{(\alpha l r)^{2 l+1}} \sum_{k=0}^{2 l}(-1)^{k} \frac{(2 l) !}{(2 l-k) !}(\alpha l r)^{2 l-k}+\frac{C}{e^{\alpha l r} r^{2 l+1}},
$$


где $C$ - произвольная постоянная. Учитывая, что $\psi(r)=r^{l} y(r)$ и меняя для удобства порядок суммирования в первом слагаемом (4.3), получим общее решение однородного уравнения (4.2):

$$
\psi_{l m}(r)=\frac{A(2 l) !}{(\alpha l)^{2 l+1}} \frac{1}{r^{l+1}} \sum_{k=0}^{2 l}(-1)^{k} \frac{(\alpha l r)^{k}}{k !}+\frac{C e^{-\alpha l r}}{r^{l+1}} .
$$

Легко проверить непосредственной подстановкой, что функция $-\eta_{l m} r^{l}$ является частным решением неоднородного уравнения (4.1). Поэтому, учитывая (4.4), выпишем общее решение уравнения (4.1):

$$
\psi_{l m}(r)=\frac{A(2 l) !}{(\alpha l)^{2 l+1}} \frac{1}{r^{l+1}} \sum_{k=0}^{2 l}(-1)^{k} \frac{(\alpha l r)^{k}}{k !}+\frac{C e^{-\alpha l r}}{r^{l+1}}-\eta_{l m} r^{l}
$$

где $A$ и $C$ - произвольные постоянные.

По смыслу задачи нас интересуют только такие решения в (4.5), которые являются ограниченными в нуле (т. е. верно неравенство $\lim _{r \rightarrow 0+0} \psi_{l m}(r)<\infty$ ), что накладывает определенные условия на значения постоянных $A$ и $C$. Действительно, если, используя разложение экспоненты в ряд Маклорена, записать (4.5) в виде

$$
\psi_{l m}(r)=\frac{1}{r^{l+1}}\left\{\left(\frac{A(2 l) !}{(\alpha l)^{2 l+1}}+C\right) \sum_{k=0}^{2 l}(-1)^{k} \frac{(\alpha l r)^{k}}{k !}+C \sum_{k=2 l+1}^{\infty}(-1)^{k} \frac{(\alpha l r)^{k}}{k !}\right\}-\eta_{l m} r^{l}
$$

легко видеть, что ограниченное в нуле решение возможно, только если выражение в больших круглых скобках обратится в нуль, т. е. при $A=-C(\alpha l)^{2 l+1} /(2 l)$ !. Подставляя это выражение в (4.5), получим общий вид ограниченного в нуле решения уравнения (4.1):

$$
\psi_{l m}(r)=C F_{l}(r)-\eta_{l m} r^{l},
$$

где $C$ - произвольная постоянная, а

$$
F_{l}(r):=\frac{1}{r^{l+1}}\left(e^{-\alpha l r}-\sum_{k=0}^{2 l}(-1)^{k} \frac{(\alpha l r)^{k}}{k !}\right)=\frac{1}{r^{l+1}} \sum_{k=2 l+1}^{\infty}(-1)^{k} \frac{(\alpha l r)^{k}}{k !} .
$$

Параметры $C$ и $\eta_{l m}$ в (4.6) легко определяются из двух условий во второй и третьей строках (3.18), после чего можно вывести следующие формулы для искомого набора чисел $\left\{\eta_{l m}\right\}$ и функций $\left\{\psi_{l m}(r)\right\}$ :

$$
\begin{gathered}
\eta_{l m}=\frac{(2 l+1) \eta_{l m}^{0}}{\mu^{0} E_{l}+l+1}, \\
\psi_{l m}(r)=\eta_{l m} r_{0}^{l} \frac{F_{l}(r)}{F_{l}\left(r_{0}\right)}-\eta_{l m} r^{l},
\end{gathered}
$$

где

$$
E_{l}:=\frac{r_{0} F_{l}^{\prime}\left(r_{0}\right)}{F_{l}\left(r_{0}\right)}, \quad \mu^{0}:=\mu\left(r_{0}\right)=c_{0} e^{\alpha r_{0}} .
$$

4 Теоретическая и математическая физика, т. 187, № 1, 2016 г. 
Согласно (2.17) искомое поле $\mathbf{H}^{\mathrm{i}}(\mathbf{r})=\nabla(\eta(\mathbf{r})+\psi(\mathbf{r}))$. $\mathrm{C}$ помощью соотношений $(3.1),(3.6),(3.7),(4.8)$ и (4.9) найдем выражение для функции $\eta(\mathbf{r})+\psi(\mathbf{r})$ в сферической системе координат:

$$
\begin{aligned}
\left.(\eta(\mathbf{r})+\psi(\mathbf{r}))\right|_{\mathrm{sph}} & =\eta(r, \theta, \varphi)+\psi(r, \theta, \varphi)=\sum_{l=1}^{\infty} \sum_{m=-l}^{l}\left(\eta_{l m} r^{l}+\psi_{l m}(r)\right) Y_{l m}(\theta, \varphi)= \\
& =\sum_{l=1}^{\infty} \sum_{m=-l}^{l} \eta_{l m} r_{0}^{l} \frac{F_{l}(r)}{F_{l}\left(r_{0}\right)} Y_{l m}(\theta, \varphi)= \\
& =\sum_{l=1}^{\infty} \frac{(2 l+1) r_{0}^{l}}{\left(\mu^{0} E_{l}+l+1\right) F_{l}\left(r_{0}\right)} F_{l}(r) Q_{l}(\theta, \varphi)
\end{aligned}
$$

где

$$
Q_{l}(\theta, \varphi):=\sum_{m=-l}^{l} \eta_{l m}^{0} Y_{l m}(\theta, \varphi), \quad l=1,2, \ldots .
$$

Подставляя выражение для $E_{l}$ из (4.10), имеем

$$
\left.(\eta(\mathbf{r})+\psi(\mathbf{r}))\right|_{\mathrm{sph}}=\sum_{l=1}^{\infty} c_{l} F_{l}(r) Q_{l}(\theta, \varphi),
$$

где

$$
c_{l}:=\frac{(2 l+1) r_{0}^{l}}{\mu^{0} r_{0} F_{l}^{\prime}\left(r_{0}\right)+(l+1) F_{l}\left(r_{0}\right)}, \quad l=1,2, \ldots .
$$

В результате для описываемой ситуации запишем следующую формулу решения уравнения (1.2), полученную в рамках подхода (2.17):

$$
\begin{gathered}
\mathbf{H}^{\mathrm{i}}(\mathbf{r})=\nabla A(\mathbf{r}), \quad \mathbf{r} \in \Omega, \\
\left.A(\mathbf{r})\right|_{\mathrm{sph}}=\sum_{l=1}^{\infty} c_{l} F_{l}(r) Q_{l}(\theta, \varphi),
\end{gathered}
$$

где $c_{l}, F_{l}(r), Q_{l}(\theta, \varphi)$ определены формулами $(4.7),(4.11)$ и $(4.12), \mu^{0}=c_{0} e^{\alpha r_{0}}$, а числа $\left\{\eta_{l m}^{0}\right\}$ определены соотношениями $(2.5)$ и (3.5). Вынося за скобки $F_{l}\left(r_{0}\right)$ в знаменателе формулы (4.12) и подставляя найденное выражение для $c_{l}$ в (4.13), выпишем следующую формулу для результирующего поля внутри $\Omega$ :

$$
\begin{aligned}
\mathbf{H}^{\mathrm{i}}(\mathbf{r}) & =\nabla A(\mathbf{r}), \quad r \in \Omega, \\
\left.A(\mathbf{r})\right|_{\mathrm{sph}} & =\sum_{l=1}^{\infty} \frac{(2 l+1) r_{0}^{l}}{\mu^{0} E_{l}+l+1} \frac{F_{l}(r)}{F_{l}\left(r_{0}\right)} Q_{l}(\theta, \varphi) .
\end{aligned}
$$

Отметим, что справедливость формулы (4.14) (в качестве решения уравнения (1.2)) проверена непосредственной подстановкой (4.14) в (1.2), однако соответствующие этой проверке выкладки слишком громоздки, а потому не приводятся. Поскольку, как отмечалось выше, решение уравнения (1.2) существует и единственно, выражение (4.14) и определяет это решение. 


\section{5. РЕЗУЛЬТИРУЮЩЕЕ ПОЛЕ ВНЕ МАГНЕТИКА И ПОЛЕ РЕАКЦИИ}

После получения аналитической формулы (4.14) для напряженности поля $\mathbf{H}^{\mathrm{i}}(\mathbf{r})$ внутри шара $\Omega$ появилась возможность из соотношения (1.3) вывести аналогичную формулу для напряженности поля $\mathbf{H}^{\mathrm{e}}(\mathbf{r})$ вне $\Omega$ при произвольном внешнем поле $\mathbf{H}^{0}(\mathbf{r})$. На основе выражения (2.7) формулу (1.3) можно записать в виде

$$
\mathbf{H}^{\mathrm{e}}(\mathbf{r})=\mathbf{H}^{0}(\mathbf{r})-\nabla W(\mathbf{r}), \quad \mathbf{r} \notin \Omega,
$$

где для $W(\mathbf{r})$ остается в силе представление $(2.8)$, но $\mathbf{r} \notin \Omega$, которое с учетом второго соотношения в (2.6) можно привести к виду

$$
W(\mathbf{r})=\int_{\Omega} \frac{\operatorname{div} \mathbf{H}^{\mathrm{i}}\left(\mathbf{r}^{\prime}\right)}{4 \pi\left|\mathbf{r}-\mathbf{r}^{\prime}\right|} d \mathbf{r}^{\prime}+\int_{S} \frac{\left(\mu^{0}-1\right) H_{n^{\prime}}^{\mathrm{i}}\left(\mathbf{r}^{\prime}\right)}{4 \pi\left|\mathbf{r}-\mathbf{r}^{\prime}\right|} d \mathbf{r}^{\prime} .
$$

Подставляя в это выражение равенство $\mathbf{H}^{\mathrm{i}}(\mathbf{r})=\nabla A(\mathbf{r})$, согласно (4.14) имеем

$$
W(\mathbf{r})=\int_{\Omega} \frac{\Delta A\left(\mathbf{r}^{\prime}\right)}{4 \pi\left|\mathbf{r}-\mathbf{r}^{\prime}\right|} d \mathbf{r}^{\prime}+\left(\mu^{0}-1\right) \int_{S} \frac{\partial A\left(\mathbf{r}^{\prime}\right)}{\partial n^{\prime}} \frac{1}{4 \pi\left|\mathbf{r}-\mathbf{r}^{\prime}\right|} d \mathbf{r}^{\prime} .
$$

Применяя основную формулу Грина (см., например, [14]), с учетом того, что $\mathbf{r} \notin \Omega$, найдем для первого слагаемого справа в (5.2) следующее представление:

$$
\int_{\Omega} \frac{\Delta A\left(\mathbf{r}^{\prime}\right)}{4 \pi\left|\mathbf{r}-\mathbf{r}^{\prime}\right|} d \mathbf{r}^{\prime}=\int_{S} \frac{\partial A\left(\mathbf{r}^{\prime}\right)}{\partial n^{\prime}} \frac{1}{4 \pi\left|\mathbf{r}-\mathbf{r}^{\prime}\right|} d \mathbf{r}^{\prime}-\int_{S} A\left(\mathbf{r}^{\prime}\right) \frac{\partial}{\partial n^{\prime}} \frac{1}{4 \pi\left|\mathbf{r}-\mathbf{r}^{\prime}\right|} d \mathbf{r}^{\prime} .
$$

Подставляя это выражение в (5.2), определим, что

$$
W(\mathbf{r})=\mu^{0} \int_{S} \frac{\partial A\left(\mathbf{r}^{\prime}\right)}{\partial n^{\prime}} \frac{1}{4 \pi\left|\mathbf{r}-\mathbf{r}^{\prime}\right|} d \mathbf{r}^{\prime}-\int_{S} A\left(\mathbf{r}^{\prime}\right) \frac{\partial}{\partial n^{\prime}} \frac{1}{4 \pi\left|\mathbf{r}-\mathbf{r}^{\prime}\right|} d \mathbf{r}^{\prime} .
$$

Найдем выражение для $W(\mathbf{r})$ в сферических координатах, переходя к ним в каждом слагаемом в правой части (5.3) по отдельности. Сначала перейдем к сферическим координатам в первом интеграле по $\mathbf{r}$, одновременно делая сферическую замену переменных в интеграле и используя выражение для $\left.A(\mathbf{r})\right|_{\mathrm{sph}}$ в (4.13) и разложение (3.4) с учетом (3.3):

$$
\begin{aligned}
\left(\int_{S} \frac{\partial A\left(\mathbf{r}^{\prime}\right)}{\partial n^{\prime}}\right. & \left.\cdot \frac{1}{4 \pi\left|\mathbf{r}-\mathbf{r}^{\prime}\right|} d \mathbf{r}^{\prime}\right)\left.\right|_{\mathrm{sph}}= \\
= & \left.\left.r_{0}^{2} \int_{0}^{2 \pi} \int_{0}^{\pi}\left(\frac{\partial}{\partial \mathbf{r}^{\prime}} A(\mathbf{r})\right)\right|_{\mathrm{sph}}\left(\frac{1}{4 \pi\left|\mathbf{r}-\mathbf{r}^{\prime}\right|}\right)\right|_{\mathrm{sph}} \sin \theta^{\prime} d \theta^{\prime} d \varphi^{\prime}= \\
= & r_{0}^{2} \int_{0}^{2 \pi} \int_{0}^{\pi} \sum_{l=1}^{\infty} \sum_{m=-l}^{l} c_{l} F_{l}^{\prime}\left(r_{0}\right) \eta_{l m}^{0} Y_{l m}\left(\theta^{\prime}, \varphi^{\prime}\right) \times \\
& \times \sum_{l=0}^{\infty} \sum_{m=-l}^{l} \frac{1}{2 l+1} \frac{r_{0}^{l}}{r^{l+1}} Y_{l m}(\theta, \varphi) Y_{l m}^{*}\left(\theta^{\prime}, \varphi^{\prime}\right) \sin \theta^{\prime} d \theta^{\prime} d \varphi^{\prime}= \\
= & r_{0}^{2} \sum_{l=1}^{\infty} \sum_{m=-l}^{l} \frac{c_{l}}{2 l+1} \frac{r_{0}^{l}}{r^{l+1}} F_{l}^{\prime}\left(r_{0}\right) \eta_{l m}^{0} Y_{l m}(\theta, \varphi) .
\end{aligned}
$$


С учетом (4.11) имеем

$$
\left.\left(\int_{S} \frac{\partial A\left(\mathbf{r}^{\prime}\right)}{\partial n^{\prime}} \frac{1}{4 \pi\left|\mathbf{r}-\mathbf{r}^{\prime}\right|} d \mathbf{r}^{\prime}\right)\right|_{\mathrm{sph}}=\sum_{l=1}^{\infty} \frac{c_{l}}{2 l+1} \frac{r_{0}^{l+2}}{r^{l+1}} F_{l}^{\prime}\left(r_{0}\right) Q_{l}(\theta, \varphi) .
$$

Для преобразования второго интеграла в (5.3) сначала найдем с учетом (3.4), что

$$
\begin{aligned}
\left.\left(\frac{\partial}{\partial n^{\prime}} \frac{1}{4 \pi\left|\mathbf{r}-\mathbf{r}^{\prime}\right|}\right)\right|_{\mathrm{sph}} & =\frac{\partial}{\partial r^{\prime}}\left(\sum_{l=0}^{\infty} \frac{1}{2 l+1} \frac{r^{\prime l}}{r^{l+1}} \sum_{m=-l}^{l} Y_{l m}(\theta, \varphi) Y_{l m}^{*}\left(\theta^{\prime}, \varphi^{\prime}\right)\right)= \\
& =\sum_{l=1}^{\infty} \frac{l}{2 l+1} \frac{r^{l-1}}{r^{l+1}} \sum_{m=-l}^{l} Y_{l m}(\theta, \varphi) Y_{l m}^{*}\left(\theta^{\prime}, \varphi^{\prime}\right) .
\end{aligned}
$$

Поэтому для второго интеграла в (5.3) с учетом (3.3) и (4.13) определим, что

$$
\begin{aligned}
\left(\int_{S} A\left(\mathbf{r}^{\prime}\right) \frac{\partial}{\partial n^{\prime}}\right. & \left.\frac{1}{4 \pi\left|\mathbf{r}-\mathbf{r}^{\prime}\right|} d \mathbf{r}^{\prime}\right)\left.\right|_{\mathrm{sph}}=r_{0}^{2} \int_{0}^{2 \pi} \int_{0}^{\pi}\left(\sum_{l=1}^{\infty} \sum_{m=-l}^{l} c_{l} F_{l}\left(r_{0}\right) \eta_{l m}^{0} Y_{l m}\left(\theta^{\prime}, \varphi^{\prime}\right)\right) \times \\
& \times\left(\sum_{l=1}^{\infty} \sum_{m=-l}^{l} \frac{l}{2 l+1} \frac{r_{0}^{l-1}}{r^{l+1}} Y_{l m}(\theta, \varphi) Y_{l m}^{*}\left(\theta^{\prime}, \varphi^{\prime}\right)\right) \sin \theta^{\prime} d \theta^{\prime} d \varphi^{\prime}= \\
= & \sum_{l=1}^{\infty} \frac{l c_{l}}{2 l+1} F_{l}\left(r_{0}\right) \frac{r_{0}^{l+1}}{r^{l+1}} Q_{l}(\theta, \varphi) .
\end{aligned}
$$

Подставляя этот интеграл и (5.4) в (5.3), выведем выражение для $W(\mathbf{r})$ в $(5.1)$ :

$$
\left.W(\mathbf{r})\right|_{\mathrm{sph}}=\sum_{l=1}^{\infty} \frac{c_{l}}{2 l+1} \frac{r_{0}^{l+1}}{r^{l+1}}\left(\mu^{0} r_{0} F_{l}^{\prime}\left(r_{0}\right)-l F_{l}\left(r_{0}\right)\right) Q_{l}(\theta, \varphi) .
$$

Подставляя сюда выражение для $c_{l}$ из (4.12) и поделив числитель и знаменатель получившейся под знаком суммы дроби на $F_{l}\left(r_{0}\right)$, имеем с учетом (4.10), что

$$
\left.W(\mathbf{r})\right|_{\mathrm{sph}}=\sum_{l=1}^{\infty} \frac{\left(\mu^{0} E_{l}-l\right) r_{0}^{2 l+1}}{\mu^{0} E_{l}+l+1} \frac{1}{r^{l+1}} Q_{l}(\theta, \varphi) .
$$

Согласно $(2.5),(3.5)$ и (4.11) внешнее поле можно представить в виде

$$
\mathbf{H}^{0}(\mathbf{r})=\nabla \eta^{0}(\mathbf{r}),\left.\quad \eta^{0}(\mathbf{r})\right|_{\mathrm{sph}}=\sum_{l=1}^{\infty} r^{l} Q_{l}(\theta, \varphi) .
$$

Тогда на основе (5.1) с учетом (5.5) и (5.6) запишем окончательную формулу для результирующего поля вне магнетика:

$$
\begin{aligned}
\mathbf{H}^{\mathrm{e}}(\mathbf{r}) & =\nabla B(\mathbf{r}), \quad \mathbf{r} \notin \Omega, \\
\left.B(\mathbf{r})\right|_{\mathrm{sph}} & =\sum_{l=1}^{\infty}\left(r^{l}-\frac{\left(\mu^{0} E_{l}-l\right) r_{0}^{2 l+1}}{\mu^{0} E_{l}+l+1} \frac{1}{r^{l+1}}\right) Q_{l}(\theta, \varphi),
\end{aligned}
$$

где $Q_{l}(\theta, \varphi), E_{l}$ и $\mu^{0}$ определены в $(4.10)$ и (4.11). 
Еще одной проверкой правильности формул (4.14) и (5.7) является тот факт, что напряженности определяемых ими полей $\mathbf{H}^{\mathrm{i}}(\mathbf{r})$ и $\mathbf{H}^{\mathrm{e}}(\mathbf{r})$ внутри и вне $\Omega$ удовлетворяют соотношению $\left.H_{n}^{\mathrm{e}}\right|_{S}=\left.\mu^{0} H_{n}^{\mathrm{i}}\right|_{S}$, связывающему нормальные составляющие этих полей на границе магнетика $S$. Выполнение этого соотношения является одним из необходимых свойств решения общего уравнения (1.1) и отражает непрерывность нормальной составляющей магнитной индукции при переходе через границу магнетиков с разными магнитными проницаемостями.

При решении практических прямых и обратных задач магнитостатики важную роль играет (и может измеряться в эксперименте) напряженность $\mathbf{H}^{\mathrm{R}}(\mathbf{r})$ так называемого поля реакции, которая показывает величину возмущения напряженности магнитного поля внешних источников в пространстве при внесении в него магнетика. Данная напряженность определяется формулой

$$
\mathbf{H}^{\mathrm{R}}(\mathbf{r})=\mathbf{H}^{\mathrm{e}}(\mathbf{r})-\mathbf{H}^{0}(\mathbf{r}), \quad \mathbf{r} \notin \Omega,
$$

откуда следует, что

$$
\mathbf{H}^{\mathrm{e}}(\mathbf{r})=\mathbf{H}^{0}(\mathbf{r})+\mathbf{H}^{\mathrm{R}}(\mathbf{r}) .
$$

Сравнение формул (5.1) и (5.9) дает равенство $\mathbf{H}^{\mathrm{R}}(\mathbf{r})=-\nabla W(\mathbf{r})$, а потому с учетом (5.5) имеем следующую формулу для напряженности поля реакции:

$$
\begin{aligned}
\mathbf{H}^{\mathrm{R}}(\mathbf{r}) & =\nabla D(\mathbf{r}), \quad \mathbf{r} \notin \Omega, \\
\left.D(\mathbf{r})\right|_{\mathrm{sph}} & =-\sum_{l=1}^{\infty} \frac{\left(\mu^{0} E_{l}-l\right) r_{0}^{2 l+1}}{\mu^{0} E_{l}+l+1} \frac{1}{r^{l+1}} Q_{l}(\theta, \varphi) .
\end{aligned}
$$

Зависимость напряженностей внешнего результирующего поля $\mathbf{H}^{\mathrm{e}}(\mathbf{r})$ и поля реакции $\mathbf{H}^{\mathrm{R}}(\mathbf{r})$ от параметров $c_{0}$ и $\alpha$ магнитной проницаемости в формулах (5.7) и (5.10) содержится во фрагменте $\mu^{0} E_{l}$ этих формул. Если воспользоваться формулами (4.10), определяющими $\mu^{0}$ и $E_{l}$, после ряда преобразований придем к явной зависимости $\mu^{0} E_{l}$ от параметров магнитной проницаемости:

$$
\mu^{0} E_{l}=-c_{0} e^{\alpha r_{0}}\left(\alpha l r_{0}+\frac{\left(\alpha l r_{0}\right)^{2 l+1} /(2 l) !}{e^{-\alpha l r_{0}}-\sum_{k=0}^{2 l}(-1)^{k}\left(\alpha l r_{0}\right)^{k} / k !}+l+1\right) .
$$

Эту формулу можно использовать не только для решения прямой задачи вычисления результирующего поля и поля реакции по формулам (5.7) и (5.10), но и для исследования обратной задачи - возможности восстановления параметров магнитной проницаемости по измеренному полю реакции или результирующему полю вне магнетика.

\section{6. СЛУЧАЙ ПОСТОЯННОЙ МАГНИТНОЙ ПРОНИЦАЕМОСТИ ИЛИ ПОСТОЯННОГО ВНЕШНЕГО ПОЛЯ}

Рассмотрим некоторые часто встречающиеся частные случаи выведенных выше формул напряженности результирующего поля внутри и вне шарового магнетика. Выбранная двухпараметрическая модель магнитной проницаемости $\mu(r)=c_{0} e^{\alpha r}$ содержит в себе и случай (в качестве предельного при $\alpha \rightarrow 0$ ) постоянной магнитной проницаемости $\mu(r)=c_{0}$. Поэтому, перейдя к такому пределу в формулах (4.14), 
(5.7) и (5.10), выпишем формулы для результирующих полей и поля реакции для случая постоянной магнитной проницаемости $\mu(r)=c_{0}$. Предварительно заметим, что из формулы (4.7) можно получить, что при $\alpha \rightarrow 0$

$$
F_{l}(r)=-\frac{l^{2 l+1} r^{l}}{(2 l+1) !} \alpha^{2 l+1}+O\left(\alpha^{2 l+2}\right), \quad F_{l}^{\prime}(r)=-\frac{l^{2 l+2} r^{l-1}}{(2 l+1) !} \alpha^{2 l+1}+O\left(\alpha^{2 l+2}\right),
$$

а потому из (4.10) и (4.7) следует, что

$$
\lim _{\alpha \rightarrow 0} E_{l}=l, \quad \lim _{\alpha \rightarrow 0}=\frac{F_{l}(r)}{F_{l}\left(r_{0}\right)}=\frac{r^{l}}{r_{0}^{l}} .
$$

Переходя к пределу при $\alpha \rightarrow 0$ в (4.14), с учетом (6.1) имеем формулу для результирующего поля внутри шарового магнетика с постоянной магнитной проницаемостью $\mu(r)=c_{0}$, помещенного в произвольное внешнее поле $\mathbf{H}^{0}(\mathbf{r})$ :

$$
\begin{aligned}
\mathbf{H}^{\mathrm{i}}(\mathbf{r}) & =\nabla A(\mathbf{r}), \quad \mathbf{r} \in \Omega \\
\left.A(\mathbf{r})\right|_{\mathrm{sph}} & =\sum_{l=1}^{\infty} \frac{2 l+1}{c_{0} l+l+1} r^{l} Q_{l}(\theta, \varphi) .
\end{aligned}
$$

Аналогично, переходя к пределу при $\alpha \rightarrow 0$ в формулах (5.7) и (5.10), с учетом (6.1) получим формулы для поля реакции и результирующего поля вне шарового магнетика с постоянной магнитной проницаемостью $\mu(r)=c_{0}$ :

$$
\begin{aligned}
\mathbf{H}^{\mathrm{e}}(\mathbf{r}) & =\nabla B(\mathbf{r}), \quad \mathbf{r} \notin \Omega, \\
\left.B(\mathbf{r})\right|_{\mathrm{sph}} & =\sum_{l=1}^{\infty}\left(r^{l}-\frac{l\left(c_{0}-1\right) r_{0}^{2 l+1}}{c_{0} l+l+1} \frac{1}{r^{l+1}}\right) Q_{l}(\theta, \varphi), \\
\mathbf{H}^{\mathrm{R}}(\mathbf{r}) & =\nabla D(\mathbf{r}), \quad \mathbf{r} \notin \Omega, \\
\left.D(\mathbf{r})\right|_{\mathrm{sph}} & =-\sum_{l=1}^{\infty} \frac{l\left(c_{0}-1\right) r_{0}^{2 l+1}}{c_{0} l+l+1} \frac{1}{r^{l+1}} Q_{l}(\theta, \varphi) .
\end{aligned}
$$

Рассмотрим случай постоянного внешнего поля $\mathbf{H}^{0}(\mathbf{r})$, величину которого обозначим как $H_{0}:=\left|\mathbf{H}^{0}(\mathbf{r})\right|$. Если ось $z$ направить вдоль этого поля, в этой системе координат $\mathbf{H}^{0}(\mathbf{r})=\left\{0,0, H_{0}\right\}$. Тогда в формуле $(2.5) \eta^{0}(\mathbf{r})=H_{0} z$, а потому

$$
\eta^{0}(r, \theta, \varphi):=\left.\eta^{0}(\mathbf{r})\right|_{\mathrm{sph}}=H_{0} r \cos \theta=\sqrt{\frac{4 \pi}{3}} H_{0} r Y_{10}(\theta, \varphi)
$$

Из выражения (3.5) следует, что описанному случаю соответствует такой набор параметров: $\eta_{10}^{0}=\sqrt{4 \pi / 3} H_{0}$, а остальные $\left\{\eta_{l m}^{0}\right\}$ равны нулю. Поэтому из формулы (4.11) выведем, что $Q_{1}(\theta, \varphi)=H_{0} \cos \theta, Q_{l}(\theta, \varphi)=0, l=2,3, \ldots$ Подставляя эти выражения в формулы (4.14), (5.7) и (5.10), найдем формулы для напряженностей результирующих полей и поля реакции в случае постоянного внешнего магнитного 
поля:

$$
\begin{aligned}
\mathbf{H}^{\mathrm{i}}(\mathbf{r}) & =\nabla A(\mathbf{r}), \quad \mathbf{r} \in \Omega, \\
\left.A(\mathbf{r})\right|_{\mathrm{sph}} & =\frac{3 r_{0} H_{0}}{\left(\mu^{0} E_{1}+2\right) F_{1}\left(r_{0}\right)} F_{1}(r) \cos \theta, \\
\mathbf{H}^{\mathrm{e}}(\mathbf{r}) & =\nabla B(\mathbf{r}), \quad \mathbf{r} \notin \Omega, \\
\left.B(\mathbf{r})\right|_{\mathrm{sph}} & =H_{0}\left(r-\frac{\left(\mu^{0} E_{1}-1\right) r_{0}^{3}}{\mu^{0} E_{1}+2} \frac{1}{r^{2}}\right) \cos \theta, \\
\mathbf{H}^{\mathrm{R}}(\mathbf{r}) & =\nabla D(\mathbf{r}), \quad \mathbf{r} \notin \Omega, \\
\left.D(\mathbf{r})\right|_{\mathrm{sph}} & =-H_{0} \frac{\left(\mu^{0} E_{1}-1\right) r_{0}^{3}}{\mu^{0} E_{1}+2} \frac{1}{r^{2}} \cos \theta,
\end{aligned}
$$

где $\mu^{0}, E_{1}$ и $F_{1}(r)$ определяются из формул $(4.7),(4.10)$. Если в формуле $(6.3)$ перейти к пределу $\alpha \rightarrow 0$ либо в формуле (6.2) положить $Q_{1}(\theta, \varphi)=H_{0} \cos \theta, Q_{l}(\theta, \varphi)=0$, $l=2,3, \ldots$, придем к известной формуле $\mathbf{H}^{\mathrm{i}}=\left(3 /\left(c_{0}+2\right)\right) \mathbf{H}^{0}$ для напряженности результирующего поля в шаре с постоянной магнитной проницаемостью $\mu=c_{0}$, помещенном в постоянное внешнее поле $\mathbf{H}^{0}$.

\section{Список литературы}

[1] Н. А. Хижняк, Интегральные уравнения макроскопической электродинамики, Наукова думка, Киев, 1986.

[2] В. Я. Раевский, ТМФ, 100:3 (1994), 323-331.

[3] M. J. Friedman, SIAM J. Appl. Math., 39:1 (1980), 14-20.

[4] С. Т. Толмачев, Специальные методы решения задач магнитостатики, Вища Школа, Киев, 1983.

[5] В.Я. Раевский, О свойствах квазиэрмитовых операторов и их применении к исследованию операторов теории потенциала и основного уравнения электро- и магнитостатики, 24/48(01), Ин-т физики металлов УрО РАН, Екатеринбург, 2001.

[6] В. В. Дякин, О. В. Умергалина, Дефектоскопия, 39:4 (2003), 52-66.

[7] В. В. Дякин, В.Я. Раевский, О. В. Умергалина, Ж. вычисл. матем. и матем. физ., 49:1 (2009), 178-188.

[8] В.В.Дякин, В. Я. Раевский, О.В.Кудряшова, Дефектоскопия, 45:8 (2009), 16-30.

[9] В. В. Дякин, О. В. Кудряшова, Дефектоскопия, 48:11 (2010), 41-52.

[10] В. В. Дякин, В. Я. Раевский, О.В.Кудряшова, Дефектоскопия, 45:3 (2009), 67-79.

[11] В. В. Дякин, В. Я. Раевский, О. В. Умергалина, Дефектоскопия, 44:2 (2008), 36-49.

[12] Э. Б. Быховский, Н. В. Смирнов, Тр. МИАН СССР, 59, 5-36.

[13] С. Г. Михлин, Линейные уравнения в частных производных, Высшая школа, М., 1977.

[14] А. Н. Тихонов, А. А. Самарский, Уравнения математической физики, Наука, М., 1977.

[15] Д. А. Варшалович, А. Н. Москалев, В. К. Херсонский, Квантовая теория углового момента, Наука, Л., 1975.

[16] Н. С. Кошляков, Э. Б. Глинер, М. М. Смирнов, Уравнения в частных производных математической физики, Высшая школа, М., 1970.

Поступила в редакцию 28.04.2015, после доработки 2.07.2015 